\title{
Correlação entre pressão inspiratória máxima, ventilação pulmonar e tempo de ventilação em pacientes ventilados no modo pressão de suporte
}

\section{Correlation between maximal inspiratory pressure, pulmonary ventilation and time of mechanical ventilation in pressure support ventilated patients}

\author{
Baldomero Antonio Kato da Silva'; Joyce Kellen Diniz Souza ${ }^{2}$; Daniel Martins Pereira ${ }^{3}$; Ricardo \\ Dutra Aydos $;$; Paulo de Tarso Camillo de Carvalho5; Filipe Abdalla dos Reis ${ }^{6}$ \\ ${ }^{1}$ Fisioterapeuta. Mestre em Ciências da Saúde -UnB/DF e Professor do Curso de Fisioterapia da Uniderp/MS \\ ${ }^{2}$ Fisioterapeuta -Uniderp/MS. \\ ${ }^{3}$ Fisioterapeuta. Mestre em Saúde e Desenvolvimento para a Região Centro-Oeste - UFMS e Professor do Curso de Fisioterapia \\ da Uniderp/MS. \\ ${ }^{4}$ Médico. Doutor em Técnicas Operatórias e Cirurgia Experimental - Unifesp. Coordenador do Programa de Pós-Graduação \\ Strictu Sensu em Saúde e Desenvolvimento - UFMS \\ ${ }^{5}$ Fisioterapeuta. Doutor em Ortopedia, Traumatologia e Reabilitação - USP e Professor do Curso de Fisioterapia - Uniderp. \\ ${ }^{6}$ Fisioterapeuta. Mestre em Bioengenharia - Univap e Professor do Curso de Fisioterapia da Uniderp. \\ Endereço para correspondência \\ R. Dona Ziza, 354 - Bl. 4, Apto. 22 - Tiradentes, \\ 79041-490 - Campo Grande - MS [Brasil] \\ e-mail: ftbaldock@uol.com.br
}

\section{Resumo}

Neste estudo, avalia-se a influência do tempo de permanência em ventilação mecânica sobre a pressão inspiratória máxima e o volume pulmonar de pacientes ventilados no modo pressão de suporte. Foram incluídos 12 pacientes adultos, 4 do sexo feminino e 8 do masculino, em desmame da ventilação mecânica no modo pressão de suporte. Para cada paciente foi aferida a pressão inspiratória máxima, volume minuto e corrente, e tempo de ventilação mecânica. A análise estatística foi feita por meio do coeficiente de correlação linear de Pearson, com nível de significância $\mathrm{p}<0,05$. Observou-se correlação negativa entre o tempo de ventilação mecânica, a pressão inspiratória máxima $(r=-0,7142 ; \mathrm{p}=0,009)$, tempo de ventilação mecânica e volume corrente $(r=-0,601 ; p=0,0387$. O tempo de permanência em ventilação mecânica tem influência negativa sobre a força muscular respiratória e o volume corrente em pacientes ventilados no modo pressão de suporte.

Descritores: Desmame; PiMax, ventilação pulmonar e tempo de VM; Ventilação à pressão de suporte; Ventilação mecânica; UTI.

\begin{abstract}
In this study, it is evaluated the influence of time in mechanical ventilation on maximal inspiratory pressure and pulmonary volume of pressure support ventilated patients. 12 adult patients, 4 female and 8 male, in weaning process under pressure support mode were included. Maximal inspiratory pressure, minute volume, tidal volume and time of mechanical ventilation were calculated for all patients. The statistical analysis was made by Pearson Linear Correlation Coefficient, with significant level $\mathrm{p}<0,05$. It was observed a negative correlation between time of mechanical ventilation and maximal inspiratory pressure $(\mathrm{r}=-0,7142 ; \mathrm{p}=0,009)$, and time of mechanical ventilation and tidal volume $(r=-0,601 ; p=0,0387)$. The time of mechanical ventilation has negative influence on respiratory muscular strength and tidal volume in pressure support ventilated patients.
\end{abstract}

Key words: ICU; Mechanical ventilation; Pressure support ventilation; Weaning. 


\section{Introdução}

A ventilação mecânica (VM) é um suporte ventilatório que consiste em um método auxiliar no tratamento e na manutenção da oxigenação e/ou ventilação de pacientes com insuficiência respiratória aguda ou crônica agudizada de maneira artificial, até que estes seja capaz de reassumi-las ${ }^{1,2}$.

Define-se desmame como o processo de transição da ventilação artificial para a espontânea e retirada do indivíduo, lenta ou rapidamente, para que ele possa assumir a ventilação espontânea sem a necessidade de uma prótese ventilatória $^{3,4,5}$.

A independência do ventilador ocorre quando o paciente passa pela triagem de respiração espontânea e não requer mais suporte ventilatório, e a remoção da via aérea artificial pode ocorrer quando existe a confirmação de que o tubo endotraqueal não é mais necessário ${ }^{6,7}$.

Os preditores de sucesso no desmame utilizados na prática clínica incluem análise de características demográficas, sinais subjetivos, sinais vitais, variáveis hemodinâmicas, trocas gasosas e variáveis bioquímicas ${ }^{8,9}$.

Os testes volumétricos à beira do leito têm sido freqüentemente usados e comumente servem de preditores para o desmame. Vários estudos têm sugerido que o volume minuto, a máxima pressão ventilatória gerada e a ventilação voluntária máxima podem ser utilizados nesse processo ${ }^{10}$.

O processo de desmame da prótese ventilatória envolve alterações na interação entre o paciente e o ventilador. Com esse processo, intenciona-se reduzir o nível de suporte oferecido pelo ventilador, requerendo que o paciente assuma a maior proporção do trabalho ventilatório, transferindo, dessa forma, a carga de trabalho do ventilador a ele ${ }^{11,12}$.

Dificuldades na descontinuidade do suporte ventilatório são observadas em 20 a $25 \%$ dos pacientes ventilados mecanicamente, com cerca de $40 \%$ do tempo de internação na terapia intensiva gasto nesse processo. A falha no des- mame é freqüentemente relacionada à disfunção muscular ventilatória, pois esses músculos exercem um papel essencial na determinação do desfecho da retirada da prótese ventilatória, conferindo à disfunção diafragmática induzida pelo ventilador um grande impacto na prática de cuidados em terapia intensiva ${ }^{13,14}$.

A atrofia muscular ventilatória desenvolve-se rapidamente (em 18 horas ou menos) e é significante tanto em relação ao diafragma quanto aos músculos periféricos durante o processo de ventilação controlada ${ }^{15,16}$.

O sucesso no desmame da ventilação mecânica depende da força dos músculos ventilatórios, do trabalho aplicado sobre esses músculos e da capacidade da geração de impulso pelo centro respiratório. A falha respiratória do desmame pode ser resultante de desordens desses três componentes e é possível que outras simultâneas de decréscimo da força e aumento de trabalho possam ocorrer ${ }^{10,13}$.

Em pacientes críticos, a avaliação da função muscular inspiratória é limitada à mensuração da pressão inspiratória máxima nas vias aéreas. Grande parte dos profissionais que atuam na terapia intensiva considera essa pressão uma manobra voluntária, normalmente dependente da motivação e cooperação do paciente. Não surpreende que a força muscular ventilatória não seja comumente utilizada na decisão clínica ou como um preditor do desfecho do desmame ${ }^{17}$.

A pressão inspiratória máxima é um método efetivo de avaliação da musculatura ventilatória. Ela pode ser utilizada como ferramenta diagnóstica de alto poder avaliativo e de fácil execução, como índice preditivo da mecânica do sistema respiratório e como parte de outros índices ventilatórios ${ }^{18}$.

As mensurações seriadas de pressão inspiratória máxima demonstram alterações funcionais da musculatura responsável pela inspiração durante o curso clínico, sendo um bom guia no manejo de pacientes com falhas repetidas em testes para desmame da ventilação mecânica. A excelente reprodutibilidade da mensuração da pressão inspiratória máxima e sua forte relação 
com a capacidade de sustentação da respiração espontânea sugerem que ela pode ser utilizada na monitorização da contratilidade muscular em pacientes mecanicamente ventilados ${ }^{17}$.

O objetivo deste trabalho foi avaliar a influência do tempo de permanência em ventilação mecânica sobre a pressão inspiratória máxima e o volume pulmonar de pacientes ventilados no modo pressão de suporte.

\section{Casuística e método}

Esta pesquisa foi realizada na Unidade de Terapia Intensiva Adulto, do Hospital Regional de Mato Grosso do Sul, localizado na cidade de Campo Grande-MS, entre abril e junho de 2008. Foram incluídos no estudo pacientes adultos, submetidos à ventilação mecânica invasiva, via tubo orotraqueal ou traqueostomia, em desmame da prótese ventilatória no modo pressão de suporte, cuja freqüência respiratória se mantivesse constantemente abaixo de 28 incursões por minuto. Excluíram-se os que apresentaram instabilidade clínica ou hemodinâmica, assincronia paciente-ventilador, doenças pulmonares ou torácicas obstrutivas e/ou restritivas em estágio agudo ou crônico e aqueles com alto risco de óbito.

Todos os responsáveis pelos indivíduos escolhidos foram esclarecidos sobre os procedimentos da pesquisa e assinaram Termo de Consentimento Livre e Esclarecido, segundo as Diretrizes e Normas Regulamentadoras de Pesquisa envolvendo Seres Humanos, constantes da Resolução do Conselho Nacional de Saúde no. 196/96. O protocolo de pesquisa foi aprovado pelo Comitê de Ética em Pesquisa da instituição.

A abordagem inicial foi realizada por meio de avaliação fisioterapêutica, contendo identificação, diagnóstico clínico, data de internação, quadro respiratório e parâmetros ventilatórios.

Os pacientes foram posicionados em decúbito dorsal elevado a $30^{\circ}$ e submetidos à coleta de volume minuto e do corrente, por intermédio do acoplamento de um ventilômetro de Wrigth Mark 8 (Wright Respirometer Mark 8, Ferraris
Medical Limited, England), no ramo expiratório do circuito do ventilador mecânico. Foi considerado como volume corrente o quociente entre o volume minuto e a freqüência respiratória.

A avaliação da pressão inspiratória máxima (PiMax) foi realizada por meio da desconexão do ventilador mecânico e acoplamento de um manovacuômetro aneróide MV-120 (GerAr Com. Equip. Ltda, São Paulo) na via aérea artificial. Para estimação dessa pressão em nível mais próximo ao volume residual, utilizou-se uma válvula expiratória unidirecional permitindo a expiração e ocluindo a inspiração por 20 segundos. A máxima pressão negativa, gerada pelo paciente durante esse período, foi considerada pressão inspiratória máxima. Os dados coletados (tempo de ventilação mecânica, volume minuto e o corrente e pressão inspiratória máxima) foram organizados em planilhas para posterior análise.

\section{Resultados}

As medidas das variáveis foram expressas em média \pm desvio-padrão. Os resultados foram tabulados de maneira que se observassem as características quanto ao sexo dos pacientes e à disposição dos valores de idade, peso, tempo de ventilação, pressão de suporte, pressão inspiratória máxima, volume minuto, volume corrente e freqüência respiratória.

A análise da relação entre as variáveis estudadas foi feita por meio do coeficiente de correlação linear de Pearson. Consideraram-se como estatisticamente significativas as comparações com $p \leq 0,05$. Para tabulação dos dados, usou-se planilha eletrônica Microsoft Office Excel 2007, e a análise estatística foi realizada com o programa Bioestat 4.0.

Foram avaliados 12 pacientes, com média de idade $55,7 \pm 19,8$ anos (de 25 a 89 anos), 8 (66,7\%) do sexo masculino, e 04 (33,3\%), do feminino.

O tempo médio de permanência em ventilação mecânica foi $11,8 \pm 10,3$ dias. A média da pressão inspiratória máxima foi $51,7 \pm 20,3$ 
$\mathrm{cmH}_{2} \mathrm{O}$; a do volume corrente, $525,9 \pm 132,0 \mathrm{ml}$ e a do volume minuto, $8015,8 \pm 1720,4 \mathrm{ml}$.

O coeficiente de correlação linear de Pearson mostrou correlação negativa significante entre as variáveis tempo de ventilação mecânica e pressão inspiratória máxima $(\mathrm{r}=-0,7142 ; \mathrm{p}=0,009)$, e entre tempo de ventilação mecânica e volume corrente $(r=-0,601 ; p=0,0387)$. (Figuras 1 e 2 )



Figura l: Diagrama de dispersão entre as varióveis tempo de ventilação mecônica e pressão inspiratória máxima. Na dispersão, observa-se correlação negativa significante $(\mathrm{r}=-0,7142 ; \mathrm{p}=0,009)$ entre as varióveis $(\mathrm{p}=$ $0,005) .(n=12)$

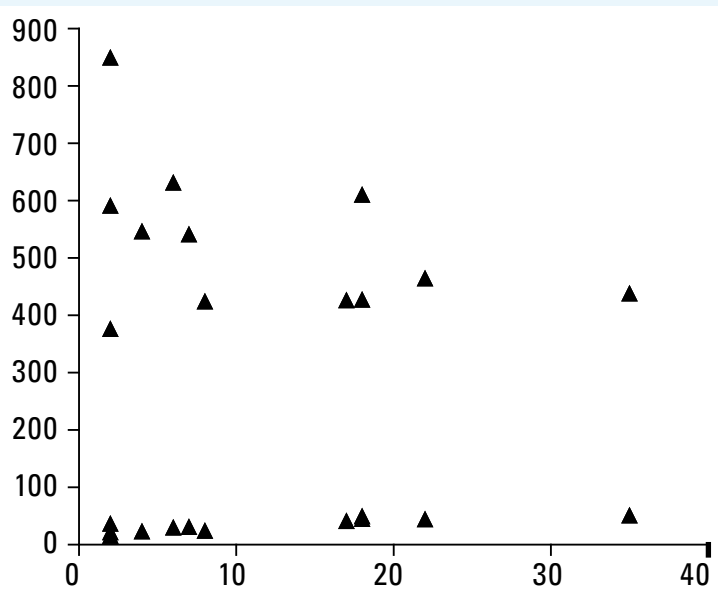

Figura 2: Diagrama de dispersão entre as varióveis tempo de ventilação mecônica e volume corrente. Na dispersão, observase correlação negativa $(r=-0,601)$ com significôncia estatística entre as variớveis $(\mathrm{p}=0,0387) .(\mathrm{n}=12)$
Observou-se ausência de correlação entre tempo de ventilação mecânica e volume minuto $(\mathrm{r}=0,1313 ; \mathrm{p}=0,6842)$. (Figura 3)

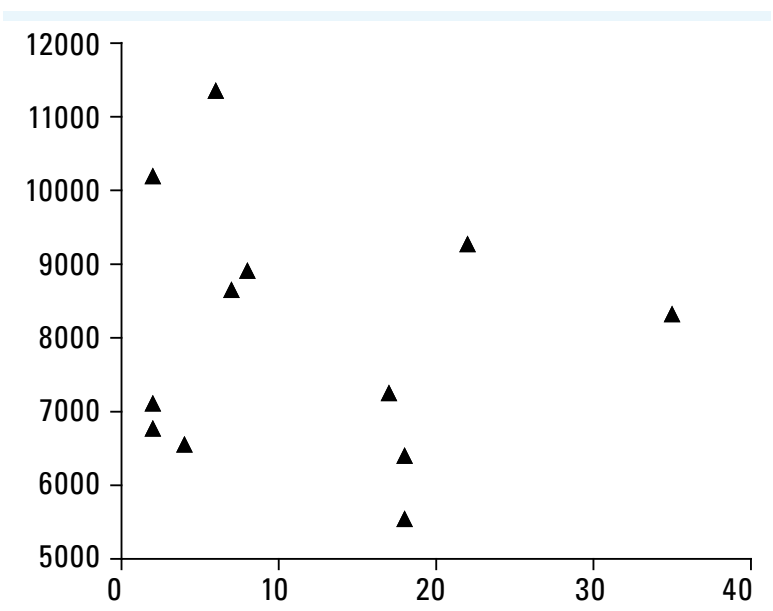

Figura 3: Diagrama de dispersão entre as varióveis tempo de ventilação mecânica e volume minuto. Na dispersão, observa-se ausência de correlação entre as varióveis ( $r$ $=0,1313 ; \mathrm{p}=0,6842)$. $(\mathrm{n}=12)$

\section{Discussão}

Entre os objetivos da ventilação mecânica inclui-se a redução do trabalho respiratório em pacientes cuja função muscular ventilatória não seja capaz de sustentá-lo ${ }^{5}$. Considerando-se a função muscular como componente responsável por gerar a bomba ventilatória durante a respiração, a proposição deste estudo foi avaliar a influência da permanência em ventilação mecânica sobre a capacidade de geração de força (e conseqüentemente de volume) pela musculatura ventilatória.

Todos os pacientes incluídos na amostra se encontravam em processo de desmame da prótese ventilatória por meio de ventilação a pressão de suporte. Não obstante esse modo ventilatório assistir o paciente durante a fase inspiratória, variáveis como volume corrente, volume minuto e freqüência respiratória ficam a cargo da capacidade do próprio paciente em gerá-los e, quando bem utilizado, pode abreviar o tempo de ventilação mecânica por 
permitir a independência precoce do suporte ventilatório ${ }^{19}$.

O tempo médio de ventilação mecânica observado neste estudo foi 11,8 dias. O tempo de ventilação considerado, a partir do qual se iniciam os efeitos deletérios nos músculos ventilatórios, varia na literatura de 48 a 72 horas $^{13,20}$.

Define-se o processo de desmame como transição da ventilação artificial para a espontânea e retirada do indivíduo, lenta ou rapidamente, para que ele possa assumir a ventilação espontânea sem a necessidade de uma prótese ventilatóriaa, ${ }^{3,5}$. Nesse contexto, a retirada precoce da prótese ventilatória e a prevenção de disfunções nos músculos respiratórios são pontos fundamentais para o sucesso do desmame.

Os preditores de sucesso no desmame utilizados na prática clínica incluem diversas características demográficas e clínicas. Os testes volumétricos à beira do leito têm sido freqüentemente usados e comumente servem de preditores para o desmame. Alguns estudos têm sugerido que o volume minuto, a máxima pressão respiratória gerada e a ventilação voluntária máxima podem ser utilizados nesse processo $^{8,10}$.

Neste estudo, buscou-se avaliar a influência do tempo de ventilação sobre as variáveis volume e força por meio da correlação entre elas. Pode-se observar significativa influência do tempo de permanência em ventilação mecânica sobre a capacidade de geração de força pelos músculos inspiratórios. Considerando-se que $25 \%$ dos pacientes incluídos encontravam-se havia apenas dois dias em ventilação mecânica, esses dados confirmam os estudos de Vassilakopoulos e Petrof, que afirmam ser um período de 48 horas tempo suficiente para o surgimento de disfunções musculares ventilatórias em pacientes ventilados mecanicamente ${ }^{13}$.

As maiores implicações clínicas das alterações diafragmáticas descritas são relacionadas a pacientes, após períodos relativamente pequenos de ventilação mecânica, nos quais a debilidade substancial do diafragma pode ocorrer e causar conseqüências adversas no processo de desmame da ventilação mecânica ${ }^{13}$.

Smina et al. estudaram 115 pacientes para verificar se a força muscular ventilatória poderia servir de preditor de sucesso no processo de extubação, após o desmame. Os autores observaram que a força muscular ventilatória dos pacientes cuja extubação culminou em insucesso (13 pacientes) era significativamente menor que a dos pacientes cujo processo de interrupção ventilatória foi realizado com êxito, sugerindo que a força da musculatura respiratória e sua capacidade na geração de volume podem ser um preditor do sucesso da extubação, morbidade e mortalidade em pacientes críticos ${ }^{6}$.

Apesar da afirmação de alguns autores sobre a necessidade de cooperação do paciente para efetuar coleta de pressão inspiratória máxima, neste estudo observaram-se facilidades na realização do referido teste, com fácil reprodutibilidade e sem intercorrências clínicas durante o procedimento $^{17,18}$.

O processo de desmame da prótese ventilatória envolve mudanças na interação entre o paciente e o ventilador. A transferência do trabalho do ventilador ao paciente exige que este último seja capaz de gerar volume corrente adequado às necessidades ventilatórias ${ }^{11}$.

$\mathrm{O}$ volume corrente médio apresentado pelos incluídos neste estudo foi $525,9 \mathrm{~mL}$, sugerindo que, a despeito da correlação negativa observada entre tempo de ventilação e volume corrente, os pacientes ainda eram capazes de gerar um volume adequado, considerando-se o volume corrente aproximado de $5 \mathrm{~mL} / \mathrm{Kg}^{3,4}$.

\section{Conclusão}

O tempo de permanência em ventilação mecânica invasiva exerce influência negativa sobre a força muscular ventilatória e o volume corrente em pacientes ventilados no modo pressão de suporte. 


\section{Referências}

1. Gonçalves LO, Cicarelli DD. Manobra de recrutamento alveolar em anestesia: como, quando e por que utilizá-la. Rev Bras Anest. 2005;55(6):631-38.

2. Barrera MAR, Ramos FM, Hernández MIV, Torres JAB, Chávez AE. Empleo de la ventilación mecânica em la unidad de cuidados intensivos. Rev Assoc Mex Med Crit Ter Int. 2002;16(5):153-58.

3. Goldwasser R, Freitas EE, Saddy F, Amado V, Okamoto V. Desmame e interrupção da ventilação mecânica. J Bras Pneumol. 2007;33(Supl 2):S128-S136.

4. José A, Dias EC, Santos VLA, Chiavone PA. Valor preditivo dos gases arteriais e índices de oxigenação no desmame da ventilação mecânica. Rev Bras Ter Inten. 2001; 13(2):50-7.

5. Azeredo CAC. Desmame do ventilador mecânico: sucesso ou insucesso? Fisioterapia Brasil. 2000;1(1):33-8.

6. Smina M, Salam A, Khamiees M, Gada P, AmoatengAdjepong Y, Manthous CA. Cough peak flows and extubations outcomes. Chest. 2003;124:262-68.

7. Tobin M. Of principles and protocols and weaning. Am J Respir Crit Care Med. 2004; 169:661-67.

8. Meade M, Guyatt G, Sinuff T, Griffith L, Hand L, Toprani G, Cook D. Trials comparing alternative weaning modes and discontinuation assessments. Chest. 2001; 120:425-37.

9. Aboussouan LS, Lattin CD, Anne VV. Determinants of time-to-weaning in a specialized respiratory care unit. Chest. 2005;128: 3117-26.

10. Goldstone J. The pulmonary physician in critical care: difficult weaning. Thorax. 2002;57:986-91.

11. Hess D. Ventilator modes used in weaning. Chest. 2001; 120:474-76
12. Namen AM, Ely EW, Tatter SB, Case LD, Lucia MA, Smith A, Landry S, Wilson JA, Glazier SS, Branch CL, Kelly DL, Bowton DL, Haponik EF. Predictor of successful extubation in neurosurgical patients. Am J Respir Crit Care Med. 2001;163:658-64.

13. Vassilakopoulos T, Petrof BJ. Ventilador-induced diaphragmatic dysfunction. Am J Respir Crit Care Med. 2004;169:336-41.

14. Krieger BP, Campos M. Weaning parameters: read the methodology before proceeding. Chest. 2002;122:1873-4.

15. Meade M, Guyatt G, Cook D, Griffith L, Sinuff T, Kergl C, Mancebo J, Esteban A, Epstein S. Prediciting success in weaning from mechanical ventilation. Chest. 2001; 120:400-24.

16. Laghi F, Tobin MJ. Disorders of the respiratory muscles. Am J Respir Crit Care Med. 2003;168:10-48.

17. Cattapan SE, Laghi F, Tobin MJ. Can diaphragmatic contractility be assessed by airway twitch pressure in mechanically ventilated patients? Thorax. 2003;58:58-62.

18. Caruso P, Friedrich C, Denari SDC, Ruiz SAL, Deheinzelin D. The unidirectional valve is the best method to determine maximal inspiratory pressure during weaning. Chest.1999;115:1096-101.

19. Carvalho CRR, Toufen Junior C, Franca SA. Ventilação mecânica: princípios, análise gráfica e modalidades ventilatórias. J Bras Pneumol. 2007;33(Supl):S54-S70.

20. Chang AT, Boots RJ, Brown MG, Paratz J, Hodges PW. Reduced inspiratory muscle endurance following successful weaning from prolonged mechanical ventilation. Chest. 2005;128:553-9. 